\title{
Numerical investigation of effect of variable viscosity on transient heat transfer over a non-isothermal axisymmetric body: nanoparticle volume fraction-dependent viscosity
}

\author{
Shobha Bagai ${ }^{1} \cdot$ Mridu Sharma $^{2}$
}

Received: 23 December 2019 / Accepted: 29 February 2020 / Published online: 12 March 2020

(c) Springer Nature Switzerland AG 2020

\begin{abstract}
The current research paper delibrates the effect of variable viscosity of the nanofluid that saturates a porous media, on transient free convection. The viscosity term that appears in the equation of conservation of momentum depends on nanoparticle volume fraction. The effect of viscosity parameter on reduced Nusselt number and reduced Sherwood number for different combinations of Buoyancy ratio $\mathrm{Nr}$, Brownian motion $\mathrm{Nb}$ and thermophoresis $\mathrm{Nt}$ is examined using the unconditionally stable Keller Box method. The conditions for existence of the numerical solution has been analytically derived. The study illustrates that the variation in viscosity has an insignificant effect on the reduced Nusselt number, but the reduced Sherwood number increases with an increase in the viscosity. Although the study can accommodate any arbitrary axisymmetric body, vertical flat plate, horizontal cylinder and sphere are three particular geometries that are considered in the paper.
\end{abstract}

Keywords Porous media - Transient free convection - Non-Newtonian nanofluid · Non-isothermal axisymmetric body . Nanoparticle-dependent viscosity $\cdot$ Keller Box method

\section{List of symbols}

\section{Greek symbols}

$\alpha \quad$ Thermal diffusivity of porous medium

$\beta \quad$ Coefficient of thermal expansion

$\delta \quad$ Grouped parameter

$\epsilon \quad$ Porosity

$\eta \quad$ Similarity variable for space component

$\gamma \quad$ Viscosity parameter

$\lambda \quad$ Wall temperature parameter

$\mu \quad$ Viscosity

$v \quad$ Kinematic viscosity

$\omega$ Rescaled nanoparticle volume fraction

$\Phi \quad$ Nanoparticle volume fraction

$\psi \quad$ Stream function

$\theta$ Dimensionless temperature $\zeta \quad$ Porosity

$t$ Similarity variable for time component

Symbols

$\kappa \quad$ Permeability

$\sigma \quad$ Heat capacity ratio

$\tau \quad$ Time component

$D_{\mathrm{B}} \quad$ Brownian diffusion coefficient

$D_{\mathrm{T}} \quad$ Thermophoretic diffusion coefficient

$f$ Dimensionless stream function

$g$ Acceleration due to gravity

Le Lewis number

$N \quad$ Non-Newtonian parameter

$\mathrm{Nb}$ Brownian motion parameter

$\mathrm{Nr}$ Buoyancy ratio parameter

Nt Thermophoresis parameter

$q \quad$ Heat flux

Shobha Bagai, shobhabagai@gmail.com | ${ }^{1}$ Cluster Innovation Center, University of Delhi, New Delhi 110007 , India. ${ }^{2}$ Department of Mathematics, University of Delhi, New Delhi, India. 
$r \quad$ Function representing wall geometry

$\mathrm{Ra}_{x} \quad$ Rayleigh number

$T \quad$ Temperature

$u, v \quad$ Velocity components in $x$-and $y$-directions

$x, y \quad$ Boundary layer coordinates

\begin{tabular}{ll}
\multicolumn{2}{l}{ Subscripts } \\
$\infty$ & Ambient condition \\
C & Cylinder \\
S & Sphere \\
v & Vertical flat plate \\
w & Wall condition
\end{tabular}

\section{Introduction}

Various types of industrial works employ the conventional heat transfer fluids such as water, glycol, oil, ethylene. In order to enhance the conductivity properties of the base fluid, nanoparticles are being added to these base fluids. The nanofluid thus obtained is assumed to be homogeneous two-component mixture. In fact, lately the nanofluid technology has made an appearance as a new technique to enhance the heat transfer. A review of the application of the nanofluid transport is given in Menni et al. [1]. Sheremet et al. [2] worked on the unsteady free convection heat transfer characteristics of nanofluid using the mathematical nanofluid model proposed by Buongiorno. An exact analysis on the effects of heat generation and nanoparticle volume concentration on an unsteady free convective flow of a nanofluid past an impulsively started infinite vertical plate is presented by Loganathan et al. [3] using Laplace transformation technique. Finite element method is used by Alsabery [4] to solve the problem of a transient free convection trapezoidal cavity filled with non-Newtonian nanofluid. The problem of one-dimensional unsteady natural convection flow past an infinite vertical cylinder with heat and mass transfer under the effect of constant heat flux at the surface of the cylinder is studied by Deka et al. [5]. A recent work on transient free convection has been carried out by Tilton [6]. He has analysed the transient diffusion which is continuously perturbed by small porosity variations that triggers nonlinear convection. Vasu et al. [7] have implemented Keller Box method on transient free convection flow of a nanofluid over a sphere. They have studied the effect of nonlinear temperature parameter on temperature and nanoparticle concentration.

Heat flux depends on viscosity, and this dependency relation is illustrated by many researchers including Lai and Kulacki [8], Pop et al. [9], Bagai [10], Jayanthi and Kumari [11], El-Amrani et al. [12], Moorthy et al. [13] and Bagai and Sharma [14]. The work cited afore takes into account the temperature-dependent viscosity. But the viscosity of the fluid also depends on nanoparticle volume fraction as discussed theoretically in review papers by Mahbubul et al. [15], Ravisankar and Tarachand [16], Rudyak and Krasnolutskii [17] and Bashirnezhad et al [18]. Noghrehabadi et al. [19] studied the effect of the nanofluid viscosity and the thermal conductivity, which are assumed as a function of local nanoparticles volume fraction, on the natural convection heat transfer over a vertical plate embedded in a porous medium saturated by a nanofluid. The effects of temperature and nanoparticles volume fraction on the viscosity of nanofluid have been investigated experimentally by Esfe et al. [20], Esfe [21], Schelfhout and Vafaei [22].

In the present study, the two-dimensional transient problem is reduced using similarity transformations to one-dimensional transient problem. The unconditionally stable Keller Box method is used to solve the system of coupled nonlinear PDEs. The effect of nanoparticle volume fraction-dependent viscosity is studied in this paper for a non-isothermal axisymmetric body immersed in a porous media saturated by nanofluids.

\section{Physical model and governing equations}

Consider a two-dimensional transient free convection problem for an axisymmetric body embedded in porous media saturated by a nanofluid. The coordinate system considered in this study is depicted in Fig. 1. The $x$-axis is along the surface of the body and $y$-axis is normal to the surface of the body.

The ambient temperature and ambient nanoparticle volume fraction are assumed to be $T_{\infty}$ and $\phi_{\infty}$, respectively. When $\tau>0$, the surface of the body is maintained at variable wall temperature $T_{\mathrm{w}}(x)$ such that $T_{\mathrm{w}}>T_{\infty}$ everywhere and surface volume fraction is maintained at constant value $\phi_{\mathrm{w}}$ such that $\phi_{\mathrm{w}}>\phi_{\infty}$. The geometric configuration function $r^{*}(x)$ is given as

$r^{*}(x)= \begin{cases}1, & \text { plane flow } \\ r(x), & \text { axisymmetric flow }\end{cases}$

and acceleration due to gravity, which is a function of geometry of the body, is given as

$g_{x}=g\left[1-\left(\frac{\mathrm{d} r}{\mathrm{~d} x}\right)^{2}\right]^{\frac{1}{2}}$

Using the boundary layer and Boussinesq approximations, the governing equations are represented by a system of coupled nonlinear PDEs and are defined as (1-4) [14]: 


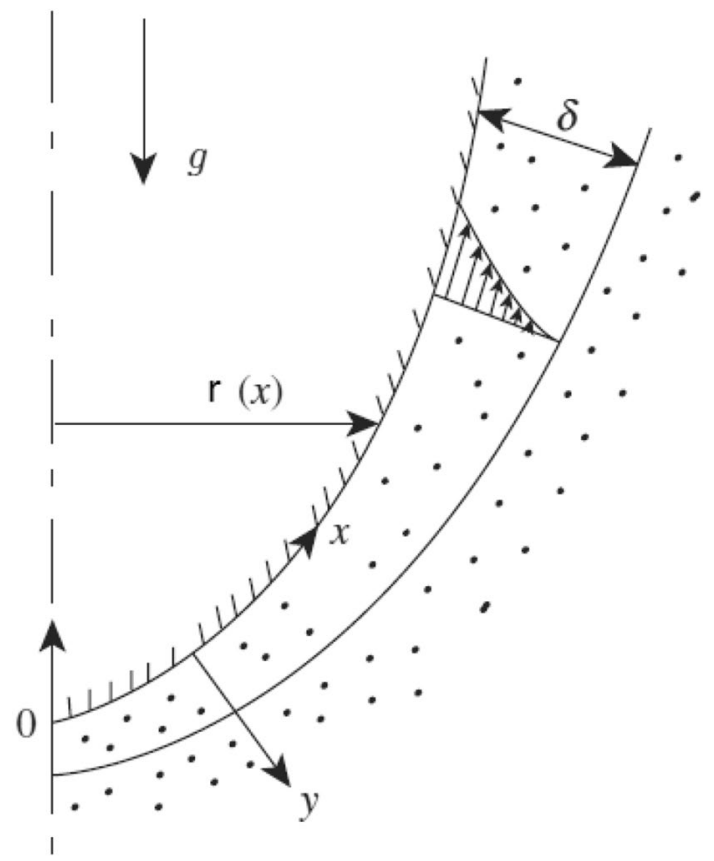

Fig. 1 Physical model

$$
\begin{aligned}
& \frac{\partial\left(r^{*} u\right)}{\partial x}+\frac{\partial\left(r^{*} v\right)}{\partial y}=0 \\
& u^{N}=\frac{\left(1-\Phi_{\infty}\right) \kappa \beta g_{x}\left(T-T_{\infty}\right)}{v_{\Phi}} \\
& \quad-\frac{\left(\rho_{p} / \rho_{f \infty}-1\right) g_{x} \kappa\left(\Phi-\Phi_{\infty}\right)}{v_{\Phi}}
\end{aligned}
$$$$
\sigma \frac{\partial T}{\partial \tau}+u \frac{\partial T}{\partial x}+v \frac{\partial T}{\partial y}=\alpha \frac{\partial^{2} T}{\partial y^{2}}
$$$$
+D_{\mathrm{B}} \frac{\partial \Phi}{\partial y} \frac{\partial T}{\partial y}+\frac{D_{\mathrm{T}}}{T_{\infty}}\left(\frac{\partial T}{\partial y}\right)^{2}
$$$$
\zeta \frac{\partial \Phi}{\partial \tau}+u \frac{\partial \Phi}{\partial x}+v \frac{\partial \Phi}{\partial y}=\epsilon\left[D_{\mathrm{B}} \frac{\partial^{2} \Phi}{\partial y^{2}}+\frac{D_{\mathrm{T}}}{T_{\infty}} \frac{\partial^{2} T}{\partial y^{2}}\right]
$$

Equation (1) represents the equation of continuity for an axisymmetric body. Equation (2) is obtained using standard order of magnitude estimation and modified Darcy's Law [23]. Equations (3) and (4) are the equation of conservation of energy and equation of conservation of nanoparticle volume fraction, respectively.

The initial and boundary conditions are given as

At $\tau=0: u=0, v=0, T=T_{\infty}$ $\tau>0: v=0, T=T_{\mathrm{w}}(x), \Phi=\Phi_{\mathrm{w}}$ at $y=0$

$u \rightarrow 0, T \rightarrow T_{\infty}, \Phi \rightarrow \Phi_{\infty}$ as $y \rightarrow \infty$

In Eq. (2), the viscosity is considered to be a function of nanoparticle volume fraction. Many researchers have suggested empirical relations between viscosity and nanoparticle volume fraction. In the beginning of the 20th century, Einstein [24] suggested a formula for the viscosity of fluid containing suspended particles. The relation suggested by him was

$\mu_{\mathrm{nf}}=\mu_{\mathrm{bf}}(1+2.5 \Phi)$

Here, $\mu_{\mathrm{nf}}$ is the viscosity of the nanofluid and $\mu_{\mathrm{bf}}$ is the viscosity of base fluid. Brinkmann [25] in the middle of the 20th century, suggested an inverse law relationship

$\mu_{\mathrm{nf}}=\frac{1}{(1-\Phi)^{2.5}} \mu_{f}$

It is interesting to note that Eq. (7) is a first-order approximation to Eq. (8). Batchelor [26] in 1977 gave another relationship between viscosity and particle volume fraction

$\mu_{\mathrm{nf}}=\left(1+2.5 \Phi+6.2 \Phi^{2}\right) \mu_{f}$

For the present study, the relation considered for the dependency of viscosity on the nanoparticle volume fraction (denoted by $v_{\Phi}$ ) is

$v_{\Phi}=v_{o}\left(1-b\left(\Phi-\Phi_{\infty}\right)\right)$

where $v_{o}$ is the viscosity at $\phi=\phi_{\infty}$.

Equation (10) is equivalent to the first-order approximation of $v_{\phi}$ considered by Noghrehabadi et al. [19]. In terms of streamfunctions $\psi, x$ and $y$ components of the velocity are defined as

$u=\frac{1}{r^{*}} \frac{\partial \psi}{\partial y}$

$v=-\frac{1}{r^{*}} \frac{\partial \psi}{\partial x}$

In order to reduce the number of independent variables involved in Eqs. (2-4), we introduce the general nondimensional transformations :

$$
\begin{aligned}
& \eta=\frac{y}{x}\left(\frac{\mathrm{Ra}_{x}}{I(x)}\right)^{\frac{1}{2}} \\
& t=\frac{\alpha}{\sigma x^{2}} \frac{\mathrm{Ra}_{x}}{I(x)} \tau
\end{aligned}
$$


$f(x, \eta, t)=\frac{\psi}{\alpha r^{*}\left(\operatorname{Ra}_{x} I(x)\right)^{\frac{1}{2}}}$

$\theta(x, \eta, t)=\frac{T-T_{\infty}}{\Delta T_{\mathrm{w}}(x)}$

$\omega(x, \eta, t)=\frac{\Phi-\Phi_{\infty}}{\Delta \Phi_{\mathrm{w}}}$

$\eta$ and $t$ are the dimensionless similarity variables in space and time variables, respectively. The modified Rayleigh number is defined by

$\mathrm{Ra}_{x}=\frac{x}{\alpha}\left(\frac{\left(1-\Phi_{\infty}\right) k \beta g_{x} \Delta T_{\mathrm{w}}}{v_{o}}\right)^{1 / N}$

The reduced form of the system of nonlinear coupled PDEs, obtained by substituting Eqs. (11-15) in Eqs. (2-4), can be written as

$$
\begin{aligned}
\left(f^{\prime}\right)^{N}=(1+\gamma \omega)\left(\theta-N r \frac{\Delta T_{\mathrm{wr}}}{\Delta T_{\mathrm{w}}(x)} \omega\right) & \\
\frac{\partial \theta}{\partial t}- & \frac{1}{2} f \theta^{\prime}+\delta f^{\prime} \theta+x I(x)\left(f^{\prime} \frac{\partial \theta}{\partial x}-\theta^{\prime} \frac{\partial f}{\partial x}\right) \\
= & \theta^{\prime \prime}+N b \theta^{\prime} \omega^{\prime}+N t \frac{\Delta T_{\mathrm{w}}(x)}{\Delta T_{\mathrm{wr}}}\left(\theta^{\prime}\right)^{2} \\
\omega^{\prime \prime}+ & \frac{N t}{N b} \frac{\Delta T_{\mathrm{w}}(x)}{\Delta T_{\mathrm{wr}}} \theta^{\prime \prime}=C \operatorname{Le} \frac{\partial \omega}{\partial t}-\frac{1}{2} \operatorname{Lef} \omega^{\prime} \\
& +x I(x) \operatorname{Le}\left(f^{\prime} \frac{\partial \omega}{\partial x}-\omega^{\prime} \frac{\partial f}{\partial x}\right)
\end{aligned}
$$

where $\gamma=b \Delta \phi_{\mathrm{w}}$, is the variable viscosity parameter which depends on nanoparticle volume fraction and the other parameters are defined as

(Buoyancy Ratio) $N r=\frac{\left(\rho_{p}-\rho_{f_{\infty}}\right) \Delta \Phi_{\mathrm{w}}}{\left(1-\Phi_{\infty}\right) \rho_{f_{\infty}} \beta \Delta T_{\mathrm{w}}}$

(Brownian Motion parameter) $N b=\frac{D_{\mathrm{B}}}{\alpha} \Delta \Phi_{\mathrm{w}}$

(Thermophoresis pramater) Nt $=\frac{D_{\mathrm{T}}}{\alpha T_{\infty}} \Delta T_{\mathrm{W}}$

(Lewis number) Le $=\frac{\alpha}{\epsilon D_{\mathrm{B}}}$
The primes in Eqs. (16-18) denote the partial derivatives with respect to $\eta$. The transformed initial and boundary conditions are given by:

At $t=0: f=0, \theta=0, \omega=0$

for $t>0: f=0, \theta=1, \omega=1 \quad$ at $\eta=0$

$$
f^{\prime} \rightarrow 0, \theta \rightarrow 0, \omega \rightarrow 0 \quad \text { as } \eta \rightarrow \infty \text {. }
$$

When the values of $x$ or $\frac{\partial f}{\partial x}$ or $\frac{\partial \theta}{\partial x}$ are small, then the equations (17) and (18) can be written as:

$$
\begin{aligned}
& \frac{\partial \theta}{\partial t}-\frac{1}{2} f \theta^{\prime}+\delta f^{\prime} \theta=\theta^{\prime \prime}+N b \theta^{\prime} \omega^{\prime} \\
& +N t \frac{\Delta T_{\mathrm{w}}(x)}{\Delta T_{\mathrm{wr}}}\left(\theta^{\prime}\right)^{2}
\end{aligned}
$$

$\omega^{\prime \prime}+\frac{N t}{N b} \frac{\Delta T_{\mathrm{w}}(x)}{\Delta T_{\mathrm{wr}}} \theta^{\prime \prime}=C \operatorname{Le} \frac{\partial \omega}{\partial t}-\frac{1}{2} \operatorname{Lef} \omega^{\prime}$

The grouped parameter $\delta$ is given by

$\delta=\frac{d \ln \Delta T_{\mathrm{w}}}{\mathrm{d} \ln x} \frac{\int_{0}^{x} r^{* 2}\left(g_{x} \Delta T_{\mathrm{w}}\right)^{\frac{1}{N}} \mathrm{~d} x}{r^{* 2}\left(g_{x} \Delta T_{\mathrm{w}}\right)^{\frac{1}{N}} x}$

Introducing new transformation variable $\xi$ as

$\xi=\int_{0}^{x} g_{x} r^{* 2} \mathrm{~d} x$

The variable $\xi$ for the case of the plane flow is defined as the distance from the lower stagnation point. It is defined as the volume segment cut by a horizontal plane for the case of an axisymmetric flow. This reduces equation (21) to

$\delta=\frac{d \ln \Delta T_{\mathrm{w}}}{\mathrm{d} \ln x} \frac{\int_{0}^{x}\left(\Delta T_{\mathrm{w}}\right)^{\frac{1}{N}} d \xi}{\left(\Delta T_{\mathrm{w}}\right)^{\frac{1}{N}} \xi}$

We define the average wall temperature as

$\Delta T_{\mathrm{wr}}=\frac{1}{\xi} \int_{0}^{\xi} \Delta T_{\mathrm{w}} d \xi$

Considering the power law profile $\Delta T_{\mathrm{w}} \propto \xi^{\lambda}$ reduces the grouped parameter to

$\delta=\frac{N \lambda}{\lambda+N}$

Thus, the final set of equations can be written as: 


$$
\begin{aligned}
& \left(f^{\prime}\right)^{N}=(1+\gamma \omega)\left(\theta-\frac{N r}{\lambda+1} \omega\right) \\
& \frac{\partial \theta}{\partial t}-\frac{1}{2} f \theta^{\prime}+\frac{N \lambda}{N+\lambda} f^{\prime} \theta=\theta^{\prime \prime}+N b \theta^{\prime} \omega^{\prime} \\
& \quad+N t(\lambda+1)\left(\theta^{\prime}\right)^{2} \\
& \omega^{\prime \prime}+\frac{N t(\lambda+1)}{N b} \theta^{\prime \prime}=C \operatorname{CL} \frac{\partial \omega}{\partial t}-\frac{1}{2} \operatorname{Le} f \omega^{\prime}
\end{aligned}
$$

\section{Solution procedure}

The system of coupled nonlinear PDEs is solved using Keller Box method. The Keller Box method is a second-order accurate finite differencing scheme in both space and time variables. It is unconditionally stable and can be used to solve coupled nonlinear PDEs. This method was originally proposed by Keller [27] and is described in a book by Cebeci and Bradshaw [28]. This method can be broadly divided into three levels. The first level is the conversion of higher-order equations to system of first-order equations. Equations (24-26) can be rewritten in the following form:

$\theta^{\prime}=\theta_{1}$

$\omega^{\prime}=\omega_{1}$

$\left(f^{\prime}\right)^{N}=(1+\gamma \omega)\left(\theta-\frac{N r}{\lambda+1} \omega\right)$

$\frac{\partial \theta}{\partial t}-\frac{1}{2} f \theta_{1}+\frac{N \lambda}{N+\lambda} f^{\prime} \theta=\theta_{1}^{\prime}+N b \omega_{1} \theta_{1}+N t(\lambda+1)\left(\theta_{1}\right)^{2}$

$\omega_{1}^{\prime}+\frac{N t(\lambda+1)}{N b} \theta_{1}^{\prime}=C \operatorname{Le} \frac{\partial \omega}{\partial t}-\frac{\operatorname{Le}}{2} f \omega_{1}$

The second level is the application of the Box scheme to the system. The first three equations are discretised about $\left(\eta_{j-1 / 2}, t^{n}\right)$ and the remaining two equations take $\left(\eta_{j-1 / 2}, t^{n-1 / 2}\right)$ as the reference point.

$$
\begin{aligned}
& \frac{\theta_{j}^{n}-\theta_{j-1}^{n}}{h}=\frac{\left(\theta_{1}\right)_{j}^{n}+\left(\theta_{1}\right)_{j-1}^{n}}{2} \\
& \frac{\omega_{j}^{n}-\omega_{j-1}^{n}}{h}=\frac{\left(\omega_{1}\right)_{j}^{n}+\left(\omega_{1}\right)_{j-1}^{n}}{2} \\
& \left(\frac{f_{j}^{n}-f_{j-1}^{n}}{h}\right)^{N}=\frac{\theta_{j}^{n}+\theta_{j-1}^{n}}{2}+\gamma \frac{\theta_{j}^{n}+\theta_{j-1}^{n}}{2} \frac{\omega_{j}^{n}+\omega_{j-1}^{n}}{2} \\
& -\frac{N r}{\lambda+1} \frac{\omega_{j}^{n}+\omega_{j-1}^{n}-\gamma \frac{N r}{\lambda+1} \frac{\left(\omega_{j}^{n}\right)^{2}+\left(\omega_{j-1}^{n}\right)^{2}}{2}}{2}
\end{aligned}
$$

The third and the final level involves linearising the discretised equations using Newton's Linearisation method.

$$
\begin{aligned}
\theta_{j}^{n+1} & =\theta_{j}^{n}+\delta \theta_{j}^{n} \\
\left(\theta_{1}\right)_{j}^{n+1} & =\left(\theta_{1}\right)_{j}^{n}+\delta\left(\theta_{1}\right)_{j}^{n} \\
\omega_{j}^{n+1} & =\omega_{j}^{n}+\delta \omega_{j}^{n} \\
\left(\omega_{1}\right)_{j}^{n+1} & =\left(\omega_{1}\right)_{j}^{n}+\delta\left(\omega_{1}\right)_{j}^{n} \\
f_{j}^{n+1} & =f_{j}^{n}+\delta f_{j}^{n}
\end{aligned}
$$

On linearisation, we obtain the final set of equations:

$$
\begin{aligned}
& -\delta \theta_{j-1}-\frac{h}{2} \delta\left(\theta_{1}\right)_{j-1}+\delta \theta_{j}-\frac{h}{2} \delta\left(\theta_{1}\right)_{j}=\left(r_{1}\right)_{j-1 / 2} \\
& -\delta \omega_{j-1}-\frac{h}{2} \delta\left(\omega_{1}\right)_{j-1}+\delta \omega_{j}-\frac{h}{2} \delta\left(\omega_{1}\right)_{j}=\left(r_{2}\right)_{j-1 / 2} \\
& \left(a_{1}\right)_{j} \delta \theta_{j-1}+\left(a_{2}\right)_{j} \delta \omega_{j-1}+\left(a_{3}\right)_{j} \delta f_{j-1}+\left(a_{1}\right)_{j} \delta \theta_{j} \\
& \quad+\left(a_{4}\right)_{j} \delta \omega_{j}-\left(a_{3}\right)_{j} \delta f_{j}=\left(r_{3}\right)_{j-1 / 2} \\
& \left(b_{1}\right)_{j} \delta \theta_{j-1}+\left(b_{2}\right)_{j} \delta\left(\theta_{1}\right)_{j-1}+\left(b_{3}\right)_{j} \delta\left(\omega_{1}\right)_{j-1} \\
& \quad+\left(b_{4}\right)_{j} \delta f_{j-1}+\left(b_{1}\right)_{j} \delta \theta_{j}+\left(b_{5}\right)_{j} \delta\left(\theta_{1}\right)_{j}+\left(b_{3}\right)_{j} \delta\left(\omega_{1}\right)_{j} \\
& +\left(b_{6}\right)_{j} \delta f_{j}=\left(r_{4}\right)_{j-1 / 2} \\
& \left(c_{1}\right)_{j} \delta\left(\theta_{1}\right)_{j-1}+\left(c_{2}\right)_{j} \delta \omega_{j-1}+\left(c_{3}\right)_{j} \delta\left(\omega_{1}\right)_{j-1} \\
& \quad+\left(c_{4}\right)_{j} \delta f_{j-1}-\left(c_{1}\right)_{j} \delta\left(\theta_{1}\right)_{j}+\left(c_{2}\right)_{j} \delta \omega_{j}+\left(c_{5}\right)_{j} \delta\left(\omega_{1}\right)_{j} \\
& +\left(c_{4}\right)_{j} \delta f_{j}=\left(r_{5}\right)_{j-1 / 2}
\end{aligned}
$$

where 


$$
\begin{aligned}
& \left(a_{1}\right)_{j}=-\frac{1}{2}-\frac{\gamma}{4}\left(\omega_{j}^{n}+\omega_{j-1}^{n}\right) \\
& \left(a_{2}\right)_{j}=\frac{N r}{2(\lambda+1)}+\frac{N r}{\lambda+1} \gamma \omega_{j-1}^{n}-\frac{\gamma}{4}\left(\theta_{j}^{n}+\theta_{j-1}^{n}\right) \\
& \left(a_{3}\right)_{j}=-\frac{N}{h^{N}}\left(f_{j}^{n}-f_{j-1}^{n}\right)^{N-1} \\
& \left(a_{4}\right)_{j}=\frac{N r}{2(\lambda+1)}+\frac{N r}{\lambda+1} \gamma \omega_{j}^{n}-\frac{\gamma}{4}\left(\theta^{n}+\theta_{j-1}^{n}\right) \\
& \left(b_{1}\right)_{j}=-\frac{N \lambda}{N+\lambda} \frac{k}{4 h}\left(f_{j}^{n-1}-f_{j-1}^{n-1}+f_{j}^{n}-f_{j-1}^{n}\right)-1 \\
& \left(b_{2}\right)_{j}=k N t(\lambda+1)\left(\theta_{1}\right)_{j-1}^{n}-\frac{k}{h}+k \frac{N b}{8}\left(\left(\omega_{1}\right)_{j}^{n-1}\right. \\
& \left.+\left(\omega_{1}\right)_{j-1}^{n-1}+\left(\omega_{1}\right)_{j}^{n}+\left(\omega_{1}\right)_{j-1}^{n}\right) \\
& +\frac{k}{16}\left(f_{j}^{n-1}+f_{j-1}^{n-1}+f_{j}^{n}+f_{j-1}^{n}\right) \\
& \left(b_{3}\right)_{j}=k \frac{N b}{8}\left(\left(\theta_{1}\right)_{j}^{n-1}+\left(\theta_{1}\right)_{j-1}^{n-1}+\left(\theta_{1}\right)_{j}^{n}+\left(\theta_{1}\right)_{j-1}^{n}\right) \\
& \left(b_{4}\right)_{j}=\frac{k}{16}\left(\left(\theta_{1}\right)_{j}^{n-1}+\left(\theta_{1}\right)_{j-1}^{n-1}+\left(\theta_{1}\right)_{j}^{n}+\left(\theta_{1}\right)_{j-1}^{n}\right) \\
& +\frac{N \lambda}{N+\lambda} \frac{k}{4 h}\left(\theta_{j}^{n-1}+\theta_{j-1}^{n-1}+\theta_{j}^{n}+\theta_{j-1}^{n}\right) \\
& \left(b_{5}\right)_{j}=k N t(\lambda+1)\left(\theta_{1}\right)_{j}^{n}+\frac{k}{h}+k \frac{N b}{8}\left(\left(\omega_{1}\right)_{j}^{n-1}\right. \\
& \left.+\left(\omega_{1}\right)_{j-1}^{n-1}+\left(\omega_{1}\right)_{j}^{n}+\left(\omega_{1}\right)_{j-1}^{n}\right) \\
& +\frac{k}{16}\left(f_{j}^{n-1}+f_{j-1}^{n-1}+f_{j}^{n}+f_{j-1}^{n}\right) \\
& \left(b_{6}\right)_{j}=\frac{k}{16}\left(\left(\theta_{1}\right)_{j}^{n-1}+\left(\theta_{1}\right)_{j-1}^{n-1}+\left(\theta_{1}\right)_{j}^{n}+\left(\theta_{1}\right)_{j-1}^{n}\right) \\
& -\frac{N \lambda}{N+\lambda} \frac{k}{4 h}\left(\theta_{j}^{n-1}+\theta_{j-1}^{n-1}+\theta_{j}^{n}+\theta_{j-1}^{n}\right) \\
& \left(c_{1}\right)_{j}=-\frac{N t(\lambda+1)}{N b} \frac{k}{h} \\
& \left(c_{2}\right)_{j}=-C L e \\
& \left(c_{3}\right)_{j}=-\frac{k}{h}+k \frac{L e}{16}\left(f_{j}^{n-1}+f_{j-1}^{n-1}+f_{j}^{n}+f_{j-1}^{n}\right) \\
& \left(c_{4}\right)_{j}=k \frac{L e}{16}\left(\left(\omega_{1}\right)_{j}^{n-1}+\left(\omega_{1}\right)_{j-1}^{n-1}+\left(\omega_{1}\right)_{j}^{n}+\left(\omega_{1}\right)_{j-1}^{n}\right) \\
& \left(c_{5}\right)_{j}=\frac{k}{h}+k \frac{L e}{16}\left(f_{j}^{n-1}+f_{j-1}^{n-1}+f_{j}^{n}+f_{j-1}^{n}\right)
\end{aligned}
$$

The boundary conditions after linearisation take the form

$\delta \theta_{0}=0 \quad \delta \omega_{0}=0$ and $\delta f_{0}=0$

$\delta \theta_{\jmath}=0 \quad \delta \omega_{\jmath}=0$

The linearised system described afore together with the modified boundary conditions takes the form $P \delta=R$ where $P$ is a block tridiagonal matrix, $\delta$ and $R$ are block column vectors.

$$
\begin{aligned}
& P=\left(\begin{array}{cccccc}
B_{1} & C_{1} & & & & \\
A_{2} & B_{2} & C_{2} & & & \\
& \ddots & \ddots & \ddots & & \\
& & \ddots & \ddots & \ddots & \\
& & & A_{J-1} & B_{J-1} & C_{J-1} \\
& & & & A_{J} & B_{J}
\end{array}\right) \\
& \delta=\left(\begin{array}{c}
\delta_{1} \\
\delta_{2} \\
\vdots \\
\delta_{J-1} \\
\delta_{J}
\end{array}\right) \text { and } R=\left(\begin{array}{c}
R_{1} \\
R_{2} \\
\vdots \\
R_{J-1} \\
R_{J}
\end{array}\right)
\end{aligned}
$$

where

$\delta_{j}=\left(\begin{array}{c}(\delta \theta)_{j} \\ \left(\delta \theta_{1}\right)_{j} \\ (\delta \omega)_{j} \\ \left(\delta \omega_{1}\right)_{j} \\ (\delta f)_{j}\end{array}\right)$ and $R_{j}=\left(\begin{array}{c}\left(r_{1}\right)_{j-1 / 2} \\ \left(r_{2}\right)_{j-1 / 2} \\ \left(r_{3}\right)_{j-1 / 2} \\ \left(r_{4}\right)_{j-1 / 2} \\ \left(r_{5}\right)_{j-1 / 2}\end{array}\right)$

The elements of $P$ are $5 \times 5$ block matrices defined as follows

$$
\begin{aligned}
& B_{1}=\left(\begin{array}{ccccc}
1 & 0 & 0 & 0 & 0 \\
-1 & -\frac{h}{2} & 0 & 0 & 0 \\
0 & 0 & 1 & 0 & 0 \\
0 & 0 & -1 & -\frac{h}{2} & 0 \\
0 & 0 & 0 & 0 & 1
\end{array}\right) \text { and }
\end{aligned}
$$

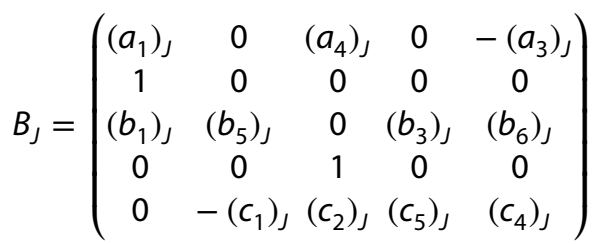

$$
\begin{aligned}
& C_{j}=\left(\begin{array}{ccccc}
0 & 0 & 0 & 0 & 0 \\
1 & -\frac{h}{2} & 0 & 0 & 0 \\
0 & 0 & 0 & 0 & 0 \\
0 & 0 & 1 & -\frac{h}{2} & 0 \\
0 & 0 & 0 & 0 & 0
\end{array}\right), j=1,2, \ldots,(J-1) \\
& A_{j}=\left(\begin{array}{ccccc}
\left(a_{1}\right)_{j} & 0 & \left(a_{2}\right)_{j} & 0 & \left(a_{3}\right)_{j} \\
0 & 0 & 0 & 0 & 0 \\
\left(b_{1}\right)_{j} & \left(b_{2}\right)_{j} & 0 & \left(b_{3}\right)_{j} & \left(b_{4}\right)_{j} \\
0 & 0 & 0 & 0 & 0 \\
0 & \left(c_{1}\right)_{j} & \left(c_{2}\right)_{j} & \left(c_{3}\right)_{j} & \left(c_{4}\right)_{j}
\end{array}\right) \text { and } \\
& B_{j}=\left(\begin{array}{ccccc}
\left(a_{1}\right)_{j} & 0 & \left(a_{4}\right)_{j} & 0 & -\left(a_{3}\right)_{j} \\
-1 & -\frac{h}{2} & 0 & 0 & 0 \\
\left(b_{1}\right)_{j} & \left(b_{5}\right)_{j} & 0 & \left(b_{3}\right)_{j} & \left(b_{6}\right)_{j} \\
0 & 0 & -1 & -\frac{h}{2} & 0 \\
0 & -\left(c_{1}\right)_{j} & \left(c_{2}\right)_{j} & \left(c_{5}\right)_{j} & \left(c_{4}\right)_{j}
\end{array}\right) \\
& \text { where } j=2,3, \ldots,(J-1) \text {. }
\end{aligned}
$$


The solution to the system of equations $P \delta=R$ exists and is unique provided $P^{-1}$ exists or in other words $P$ is non-singular. The block matrix $P$ is LU-decomposed as

$P=\mathrm{LU}$

where

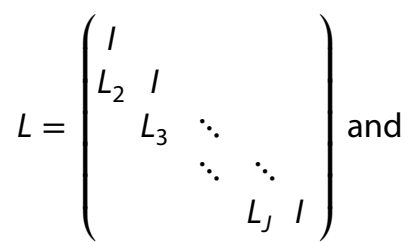

$U=\left(\begin{array}{ccccc}U_{1} & C_{1} & & & \\ & U_{2} & \ddots & & \\ & & \ddots & \ddots & \\ & & & \ddots & C_{J-1} \\ & & & & U_{J}\end{array}\right)$

The elements of the above matrix are determined recursively by the following equations:

$U_{1}=B_{1}$

$L_{j}=A_{j} U_{j-1}^{-1}$

$U_{j}=B_{j}-L_{j} C_{j-1}, j=2,3, \ldots, J$

where each $L_{j}$ and $U_{j}$ is a $5 \times 5$ matrix. The matrix $L_{j}$ is defined as:

$L_{j}=\left(\begin{array}{ccccc}\left(L_{j}\right)_{1} & \left(L_{j}\right)_{2} & \left(L_{j}\right)_{3} & \left(L_{j}\right)_{4} & \left(L_{j}\right)_{5} \\ 0 & 0 & 0 & 0 & 0 \\ \left(L_{j}\right)_{6} & \left(L_{j}\right)_{7} & \left(L_{j}\right)_{8} & \left(L_{j}\right)_{9} & \left(L_{j}\right)_{10} \\ 0 & 0 & 0 & 0 & 0 \\ \left(L_{j}\right)_{11} & \left(L_{j}\right)_{12} & \left(L_{j}\right)_{13} & \left(L_{j}\right)_{14} & \left(L_{j}\right)_{15}\end{array}\right)$

The identity matrix, $I$ appears as diagonal element of the lower triangular matrix $L$, so $\operatorname{det}(L) \neq 0$. In order to prove that $P$ is non-singular, we need to show that $U$ is also non-singular.

In order to do so, we take the basic model of an isothermal body $(\lambda=0)$, Newtonian fluid $(N=1)$ and constant viscosity $(\gamma=0)$.

For the basic model, we have

$$
\begin{aligned}
& \left(a_{1}\right)_{j}=-\frac{1}{2} \\
& \left(a_{2}\right)_{j}=\left(a_{4}\right)_{j}=\frac{N r}{2} \\
& \left(a_{3}\right)_{j}=-\frac{1}{h} \\
& \left(b_{1}\right)_{j}=-1 \\
& \left(b_{2}\right)_{j}=k N t\left(\theta_{1}\right)_{j-1}^{n}-\frac{k}{h}+k \frac{N b}{8}\left(\left(\omega_{1}\right)_{j}^{n-1}\right. \\
& \left.+\left(\omega_{1}\right)_{j-1}^{n-1}+\left(\omega_{1}\right)_{j}^{n}+\left(\omega_{1}\right)_{j-1}^{n}\right) \\
& +\frac{k}{16}\left(f_{j}^{n-1}+f_{j-1}^{n-1}+f_{j}^{n}+f_{j-1}^{n}\right) \\
& \left(b_{3}\right)_{j}=k \frac{N b}{8}\left(\left(\theta_{1}\right)_{j}^{n-1}+\left(\theta_{1}\right)_{j-1}^{n-1}+\left(\theta_{1}\right)_{j}^{n}+\left(\theta_{1}\right)_{j-1}^{n}\right) \\
& \left(b_{4}\right)_{j}=\left(b_{6}\right)_{j}=\frac{k}{16}\left(\left(\theta_{1}\right)_{j}^{n-1}+\left(\theta_{1}\right)_{j-1}^{n-1}+\left(\theta_{1}\right)_{j}^{n}+\left(\theta_{1}\right)_{j-1}^{n}\right) \\
& \left(b_{5}\right)_{j}=k N t\left(\theta_{1}\right)_{j}^{n}+\frac{k}{h}+k \frac{N b}{8}\left(\left(\omega_{1}\right)_{j}^{n-1}\right. \\
& \left.+\left(\omega_{1}\right)_{j-1}^{n-1}+\left(\omega_{1}\right)_{j}^{n}+\left(\omega_{1}\right)_{j-1}^{n}\right) \\
& +\frac{k}{16}\left(f_{j}^{n-1}+f_{j-1}^{n-1}+f_{j}^{n}+f_{j-1}^{n}\right) \\
& \left(c_{1}\right)_{j}=-\frac{N t}{N b} \frac{k}{h} \\
& \left(c_{2}\right)_{j}=-C L e \\
& \left(c_{3}\right)_{j}=-\frac{k}{h}+k \frac{L e}{16}\left(f_{j}^{n-1}+f_{j-1}^{n-1}+f_{j}^{n}+f_{j-1}^{n}\right) \\
& \left(c_{4}\right)_{j}=k \frac{L e}{16}\left(\left(\omega_{1}\right)_{j}^{n-1}+\left(\omega_{1}\right)_{j-1}^{n-1}+\left(\omega_{1}\right)_{j}^{n}+\left(\omega_{1}\right)_{j-1}^{n}\right) \\
& \left(c_{5}\right)_{j}=\frac{k}{h}+k \frac{L e}{16}\left(f_{j}^{n-1}+f_{j-1}^{n-1}+f_{j}^{n}+f_{j-1}^{n}\right) \\
& \text { For } j=1 \text {, } \\
& \operatorname{det}\left(U_{1}\right)=\operatorname{det}\left(B_{1}\right)=h^{2} / 4 \neq 0 \\
& \text { For } j=2,3, \ldots,(J-1) \\
& \operatorname{det}\left(U_{j}\right)=\operatorname{det}\left(B_{j}-L_{j} C_{j-1}\right) \\
& =\operatorname{det}\left(B_{j}+u_{j} v_{j-1}^{T}\right) \\
& \text { where } u_{j}=\left(\begin{array}{ccc}
-\left(L_{j}\right)_{2} & -\left(L_{j}\right)_{4} \\
0 & 0 \\
-\left(L_{j}\right)_{7} & -\left(L_{j}\right)_{9} \\
0 & 0 \\
-\left(L_{j}\right)_{12} & -\left(L_{j}\right)_{14}
\end{array}\right) \text { and } v_{j-1}=\left(\begin{array}{cc}
1 & 0 \\
-\frac{h}{2} & 0 \\
0 & 1 \\
0 & -\frac{h}{2} \\
0 & 0
\end{array}\right) \text {. }
\end{aligned}
$$

Using the matrix determinant lemma :

$\operatorname{det}\left(U_{j}\right)=\operatorname{det}\left(I_{2}+v_{j-1}^{T} B_{j}^{-1} u_{j}\right) \operatorname{det}\left(B_{j}\right)$

provided $B_{j}$ is non-singular. To prove that, consider 


$$
\begin{aligned}
\operatorname{det}\left(B_{j}\right)= & \frac{1}{4}\left(-4\left(a_{3}\right)_{j}\left(b_{3}\right)_{j}\left(c_{1}\right)_{j}-4\left(a_{3}\right)_{j}\left(b_{5}\right)_{j}\left(c_{5}\right)_{j}\right. \\
& +2 h\left(a_{2}\right)_{j}\left(b_{4}\right)_{j}\left(c_{1}\right)_{j}+2 h\left(a_{3}\right)_{j}\left(b_{5}\right)_{j}\left(c_{2}\right)_{j} \\
& -2 h\left(a_{1}\right)_{j}\left(b_{3}\right)_{j}\left(c_{4}\right)_{j} \\
& +2 h\left(a_{2}\right)_{j}\left(b_{5}\right)_{j}\left(c_{4}\right)_{j}+2 h\left(a_{3}\right)_{j}\left(b_{1}\right)_{j}\left(c_{5}\right)_{j} \\
& +2 h\left(a_{1}\right)_{j}\left(b_{4}\right)_{j}\left(c_{5}\right)_{j}-h^{2}\left(a_{3}\right)_{j}\left(b_{1}\right)_{j}\left(c_{2}\right)_{j} \\
& \left.-h^{2}\left(a_{1}\right)_{j}\left(b_{4}\right)_{j}\left(c_{2}\right)_{j}-h^{2}\left(a_{2}\right)_{j}\left(b_{1}\right)_{j}\left(c_{4}\right)_{j}\right)
\end{aligned}
$$

which is positive. We also have $\operatorname{det}\left(B_{J}\right)>0$. Consider

$$
\begin{aligned}
\operatorname{det}\left(I_{2}+v_{j-1}^{T} B_{j}^{-1} u_{j}\right) \\
=\frac{16\left(a_{3}\right)_{j}\left(-\left(b_{3}\right)_{j}\left(c_{1}\right)_{j}+\left(b_{2}\right)_{j}\left(c_{3}\right)_{j}\right)}{\text { Denominator }}
\end{aligned}
$$

where

\section{Denominator}

$$
\begin{aligned}
= & 4\left(a_{3}\right)_{j}\left(b_{3}\right)_{j}\left(c_{1}\right)_{j}+\left(a_{3}\right)_{j}\left[2\left(b_{5}\right)_{j}\right. \\
& \left.-h\left(b_{1}\right)_{j}\right]\left[2\left(c_{5}\right)_{j}-h\left(c_{2}\right)_{j}\right] \\
& +h\left(a_{1}\right)_{j}\left[-2\left(b_{4}\right)_{j}\left(c_{5}\right)_{j}+2\left(b_{3}\right)_{j}\left(c_{4}\right)_{j}\right. \\
& \left.+h\left(b_{4}\right)_{j}\left(c_{2}\right)_{j}\right]+h\left(a_{2}\right)_{j}\left[-2\left(b_{4}\right)_{j}\left(c_{1}\right)_{j}\right. \\
& \left.-2\left(b_{4}\right)_{j}\left(c_{5}\right)_{j}+h\left(b_{1}\right)_{j}\left(c_{4}\right)_{j}\right]
\end{aligned}
$$

$\operatorname{Det}\left(I_{2}+v_{j-1}^{T} B_{j}^{-1} u_{j}\right)$ turns out to be negative for all choices of the step length $h$ and $k$. Thus, $\operatorname{det}\left(U_{j}\right) \neq 0$ for each $j=2,3, \ldots,(J-1)$. By similar analysis, it can be shown that $\operatorname{det}\left(U_{J}\right) \neq 0$.

\section{Results and discussions}

The system (32-36) together with initial and boundary conditions is solved for selected values of viscosity parameter $\gamma$, thermophoresis parameter $N t$, Brownian motion parameter $\mathrm{Nb}$ and Buoyancy ratio parameter $\mathrm{Nr}$. In order to discretise the domain, uniform grid distribution has been used. As described in the study by Vasu et al. [7], the method is independent of grid sizes. For computation, we take the step size with respect to $\eta$ and $t$ as 0.02 and 0.01 , respectively. The value of $C$ is taken to be 0.1 . The solution is accepted when tolerance level reaches $10^{-5}$. From equation (26), it is evident that at steady state, the equation becomes independent of $C$. The effect of nanoparticle fraction-dependent viscosity on reduced Nusselt number, $-\theta^{\prime}(0)$ and reduced Sherwood number, $-\omega^{\prime}(0)$ is reflected in Table 1. The Nusselt number and Sherwood number increase with increase in viscosity parameter $\gamma$. As the viscosity of the nanofluid, $\gamma$, is a function of nanoparticle volume fraction, its effect is insignificant on reduced Nusselt number whereas the value of $-\omega^{\prime}(0)$ increases significantly as $\gamma$ increases. This trend can be verified by results obtained in Tables 2, 3 and 4. Afore mentioned tables also depicts the effect of vital parameters $\mathrm{Nt}, \mathrm{Nb}$ and $\mathrm{Nr}$ involved in the current model for isothermal and non-isothermal immersed bodies, respectively. For isothermal case, the change in reduced Nusselt number and reduced Sherwood number is insignificant on varying any of the buoyancy ratio parameter, Brownian motion parameter and thermophoresis parameter. As the range of $\mathrm{Nb}$ and $\mathrm{Nt}$ is very small, from equation (26) it is evident that

\begin{tabular}{|c|c|c|c|c|c|c|c|}
\hline \multirow[t]{2}{*}{$\lambda$} & \multirow[t]{2}{*}{$\gamma$} & \multicolumn{2}{|l|}{$N=0.5$} & \multicolumn{2}{|l|}{$N=1$} & \multicolumn{2}{|l|}{$N=2$} \\
\hline & & $-\theta^{\prime}(0)$ & $-\omega^{\prime}(0)$ & $-\theta^{\prime}(0)$ & $-\omega^{\prime}(0)$ & $-\theta^{\prime}(0)$ & $-\omega^{\prime}(0)$ \\
\hline \multirow[t]{5}{*}{0} & -0.5 & 0.3661 & 12.0304 & 0.4379 & 14.3623 & 0.4906 & 15.7159 \\
\hline & -0.1 & 0.3759 & 16.0552 & 0.4429 & 16.6092 & 0.4934 & 17.0249 \\
\hline & -0.05 & 0.3768 & 16.5449 & 0.4434 & 16.8583 & 0.4934 & 17.0249 \\
\hline & -0.005 & 0.3776 & 16.9821 & 0.4439 & 17.0782 & 0.4937 & 17.1351 \\
\hline & 0 & 0.3784 & 17.0302 & 0.4439 & 17.1023 & 0.4937 & 17.1471 \\
\hline \multirow[t]{5}{*}{1} & -0.5 & 0.5145 & 11.9487 & 0.6896 & 14.2931 & 0.8460 & 15.6623 \\
\hline & -0.1 & 0.5311 & 15.9835 & 0.7004 & 16.5437 & 0.8524 & 16.8467 \\
\hline & -0.05 & 0.5330 & 16.4741 & 0.7012 & 16.7937 & 0.8531 & 16.9728 \\
\hline & -0.005 & 0.5342 & 16.9122 & 0.7022 & 17.0139 & 0.8537 & 17.0831 \\
\hline & 0 & 0.5346 & 16.9605 & 0.7023 & 17.0381 & 0.8537 & 17.0952 \\
\hline
\end{tabular}
the Brownian motion parameter $\mathrm{Nb}$ should have an effect on the reduced Sherwood number $-\omega^{\prime}(0)$. This is verified from the values depicted in Table 3 where $-\omega^{\prime}(0)$ increases remarkably as $\mathrm{Nb}$ increases for non-isothermal case.

The values obtained by Keller Box method for Newtonian fluid for isothermal bodies are in good agreement with values given by Nogherabadi et al. [19] marked by asterisk in Table 5. Since the effect of $\gamma$ is insignificant on $-\theta^{\prime}(0)$, temperature profile doesn't mutate with changing $\gamma$. In the study conducted by Bagai and Sharma [14] where

Table 1 Effect of $\gamma$ on reduced Nusselt number $-\theta^{\prime}(0)$ and reduced Sherwood number- $\omega^{\prime}(0)$ at steady state for vertical plate $\left(\mathrm{Nt}=10^{-6}, \mathrm{Nb}=10^{-5}\right.$ and $\left.\mathrm{Nr}=10^{-3}\right)$ 
Table 2 Effect of variation of thermophoresis parameter on reduced Nusselt number $-\theta^{\prime}(0)$ and reduced Sherwood number $-\omega^{\prime}(0)$ at steady state for vertical plate $\left(\gamma=-0.5, \mathrm{Nb}=10^{-4}\right.$ and $\left.\mathrm{Nr}=10^{-2}\right)$

\begin{tabular}{|c|c|c|c|c|c|c|c|}
\hline \multirow[t]{2}{*}{$\lambda$} & \multirow[t]{2}{*}{$N t$} & \multicolumn{2}{|l|}{$N=0.5$} & \multicolumn{2}{|l|}{$N=1$} & \multicolumn{2}{|l|}{$N=2$} \\
\hline & & $-\theta^{\prime}(0)$ & $-\omega^{\prime}(0)$ & $-\theta^{\prime}(0)$ & $-\omega^{\prime}(0)$ & $-\theta^{\prime}(0)$ & $-\omega^{\prime}(0)$ \\
\hline \multirow[t]{4}{*}{0} & $10^{-9}$ & 0.3651 & 11.9722 & 0.4378 & 14.3278 & 0.4905 & 15.6978 \\
\hline & $10^{-8}$ & 0.3670 & 11.9722 & 0.4378 & 14.3277 & 0.4905 & 15.6978 \\
\hline & $10^{-7}$ & 0.3670 & 11.9722 & 0.4378 & 14.3277 & 0.4905 & 15.6978 \\
\hline & $10^{-6}$ & 0.3670 & 11.9721 & 0.4378 & 14.3276 & 0.4905 & 15.6977 \\
\hline \multirow[t]{4}{*}{1} & $10^{-9}$ & 0.5146 & 11.9279 & 0.6894 & 14.2876 & 0.8459 & 15.6683 \\
\hline & $10^{-8}$ & 0.5146 & 11.9279 & 0.6894 & 14.2876 & 0.8459 & 15.6683 \\
\hline & $10^{-7}$ & 0.5146 & 11.9278 & 0.6894 & 14.2875 & 0.8459 & 15.6682 \\
\hline & $10^{-6}$ & 0.5146 & 11.9270 & 0.6894 & 14.2864 & 0.8459 & 15.6667 \\
\hline
\end{tabular}

Table 3 Effect of variation of Brownian motion parameter on reduced Nusselt number $-\theta^{\prime}(0)$ and reduced Sherwood number $-\omega^{\prime}(0)$ at steady state for vertical plate $\left(\gamma=-0.5, \mathrm{Nt}=10^{-6}\right.$ and $\left.\mathrm{Nr}=10^{-2}\right)$

\begin{tabular}{|c|c|c|c|c|c|c|c|}
\hline \multirow[t]{2}{*}{$\lambda$} & \multirow[t]{2}{*}{$N b$} & \multicolumn{2}{|l|}{$N=0.5$} & \multicolumn{2}{|l|}{$N=1$} & \multicolumn{2}{|l|}{$N=2$} \\
\hline & & $-\theta^{\prime}(0)$ & $-\omega^{\prime}(0)$ & $-\theta^{\prime}(0)$ & $-\omega^{\prime}(0)$ & $-\theta^{\prime}(0)$ & $-\omega^{\prime}(0)$ \\
\hline \multirow[t]{4}{*}{0} & $10^{-7}$ & 0.3657 & 11.8222 & 0.4371 & 14.1702 & 0.4905 & 15.6978 \\
\hline & $10^{-6}$ & 0.3670 & 11.9722 & 0.4378 & 14.3277 & 0.4905 & 15.6978 \\
\hline & $10^{-5}$ & 0.3670 & 11.9722 & 0.4378 & 14.3277 & 0.4905 & 15.6978 \\
\hline & $10^{-4}$ & 0.3670 & 11.9721 & 0.4378 & 14.3276 & 0.4905 & 15.6977 \\
\hline \multirow[t]{4}{*}{1} & $10^{-7}$ & 0.5076 & 11.0236 & 0.6839 & 14.1608 & 0.8456 & 15.5085 \\
\hline & $10^{-6}$ & 0.5139 & 11.8364 & 0.6889 & 14.1608 & 0.8456 & 15.5085 \\
\hline & $10^{-5}$ & 0.5146 & 11.9187 & 0.6894 & 14.2749 & 0.8459 & 15.6523 \\
\hline & $10^{-4}$ & 0.5146 & 11.9269 & 0.6894 & 14.2864 & 0.8459 & 15.6667 \\
\hline
\end{tabular}

Table 4 Effect of variation of Buoyancy ratio parameter on reduced Nusselt number $-\theta^{\prime}(0)$ and reduced Sherwood number $-\omega^{\prime}(0)$ at steady state for vertical plate $\left(\gamma=-0.5, N t=10^{-6}\right.$ and $\left.N b=10^{-5}\right)$

\begin{tabular}{|c|c|c|c|c|c|c|c|}
\hline \multirow[t]{2}{*}{$\lambda$} & \multirow[t]{2}{*}{$\mathrm{Nr}$} & \multicolumn{2}{|l|}{$N=0.5$} & \multicolumn{2}{|l|}{$N=1$} & \multicolumn{2}{|l|}{$N=2$} \\
\hline & & $-\theta^{\prime}(0)$ & $-\omega^{\prime}(0)$ & $-\theta^{\prime}(0)$ & $-\omega^{\prime}(0)$ & $-\theta^{\prime}(0)$ & $-\omega^{\prime}(0)$ \\
\hline \multirow[t]{4}{*}{0} & $10^{-5}$ & 0.3672 & 12.0364 & 0.4379 & 14.3661 & 0.4906 & 15.7181 \\
\hline & $10^{-4}$ & 0.3672 & 12.0359 & 0.4379 & 14.3658 & 0.4906 & 15.7179 \\
\hline & $10^{-3}$ & 0.3672 & 12.0299 & 0.4379 & 14.3622 & 0.4906 & 15.7159 \\
\hline & $10^{-2}$ & 0.3670 & 11.9707 & 0.4378 & 14.3262 & 0.4905 & 15.6962 \\
\hline \multirow[t]{4}{*}{1} & $10^{-5}$ & 0.5148 & 11.9518 & 0.6895 & 14.2951 & 0.8460 & 15.6633 \\
\hline & $10^{-4}$ & 0.5148 & 11.9515 & 0.6895 & 14.2949 & 0.8460 & 15.6632 \\
\hline & $10^{-3}$ & 0.5148 & 11.9485 & 0.6895 & 14.2931 & 0.8460 & 15.6623 \\
\hline & $10^{-2}$ & 0.5146 & 11.9187 & 0.6894 & 14.2749 & 0.8459 & 15.6523 \\
\hline
\end{tabular}




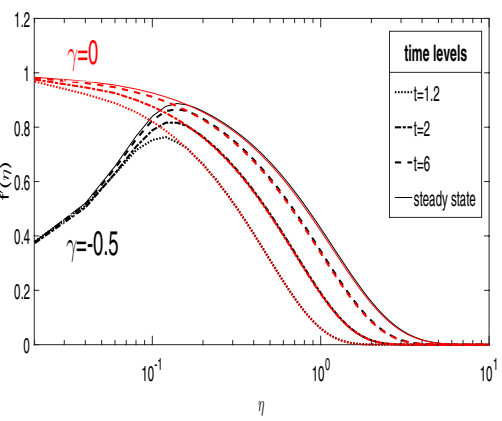

(a) Pseudoplastic fluid

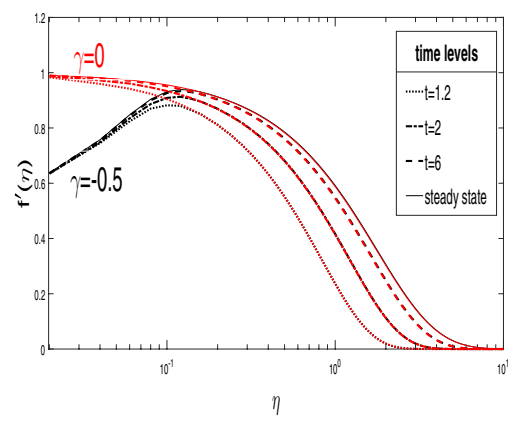

(b) Newtonian Fluid

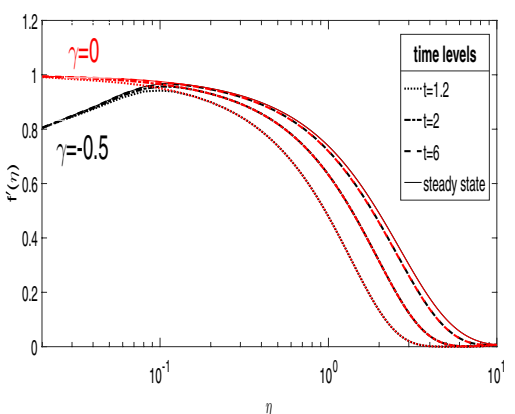

(c) Dilatant Fluid

Fig. 2 Velocity profile $\left(\mathrm{Nt}=10^{-5}, \mathrm{Nr}=10^{-2}, \mathrm{Nb}=10^{-4}\right.$ and $\left.\mathrm{Le}=10^{3}\right)$

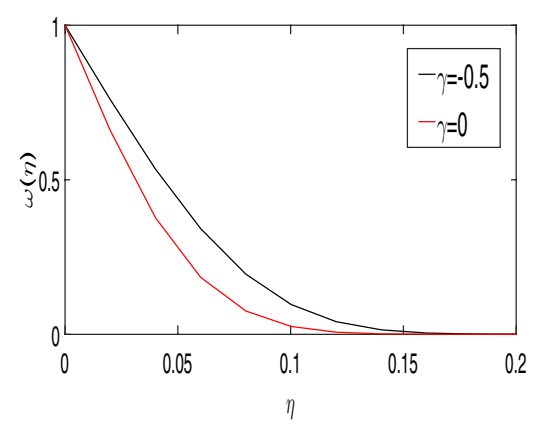

(a) Pseudoplastic fluid

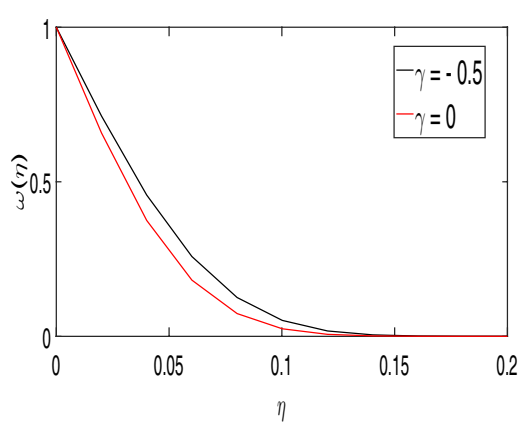

(b) Newtonian Fluid

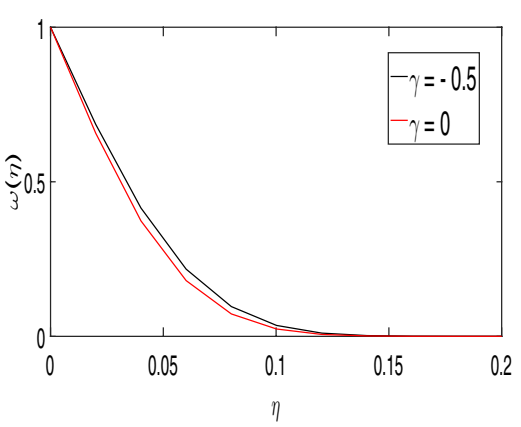

(c) Dilatant Fluid

Fig. 3 Concentration profile $\left(\mathrm{Nt}=10^{-6}, \mathrm{Nr}=10^{-3}, \mathrm{Nb}=10^{-5}\right.$ and Le $\left.=10^{3}\right)$

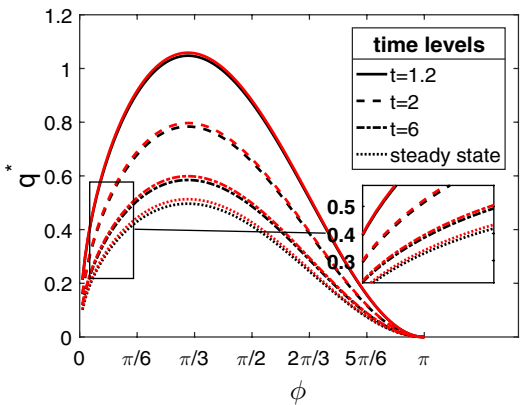

(a) Pseudoplastic fluid

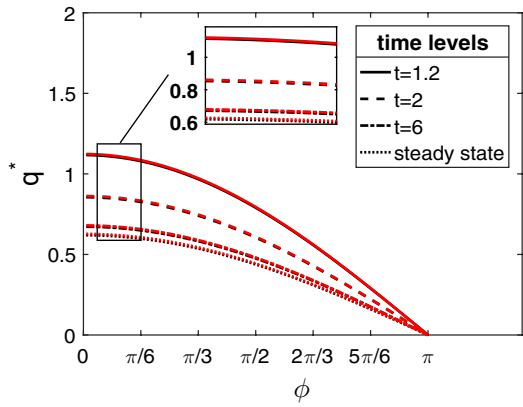

(b) Newtonian Fluid

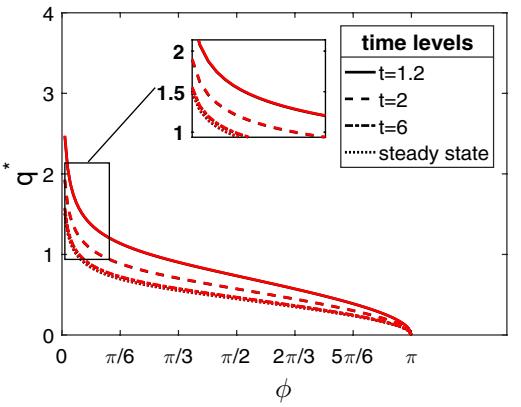

(c) Dilatant Fluid

Fig. 4 Variation of heat flux with $\phi$ for Cylinder: Isothermal case

the viscosity was considered to be dependent on temperature, the variation in $\gamma$ has a significant effect on reduced Nusselt number. The local heat flux for the case of vertical

$$
q_{v}{ }^{*}=-\theta^{\prime}(0)\left(\frac{\lambda+N}{N}\right)^{\frac{1}{2}}
$$




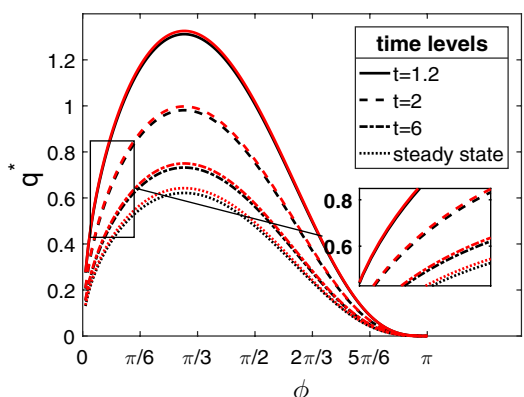

(a) Pseudoplastic fluid

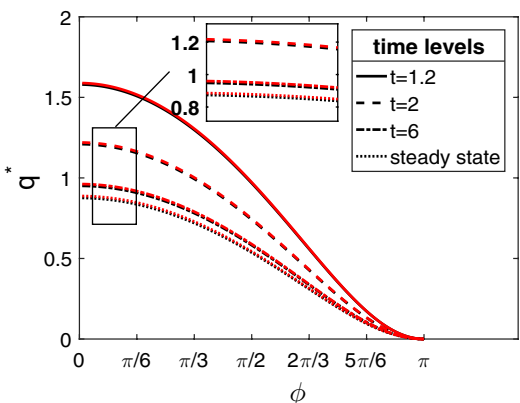

(b) Newtonian Fluid

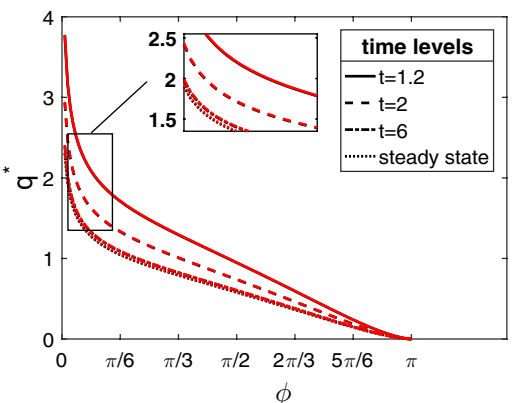

(c) Dilatant Fluid

Fig. 5 Variation of heat flux with $\phi$ for Sphere: Isothermal case

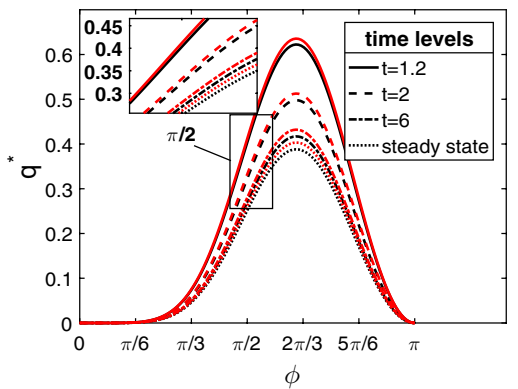

(a) Pseudoplastic Fluid

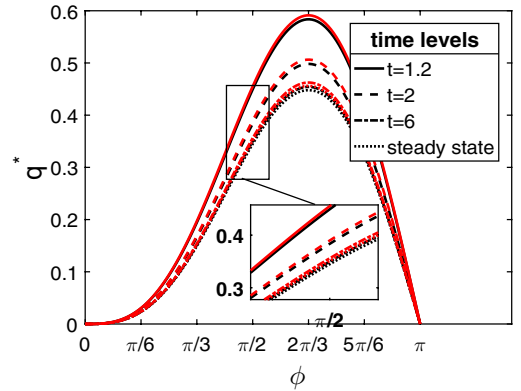

(b) Newtonian Fluid

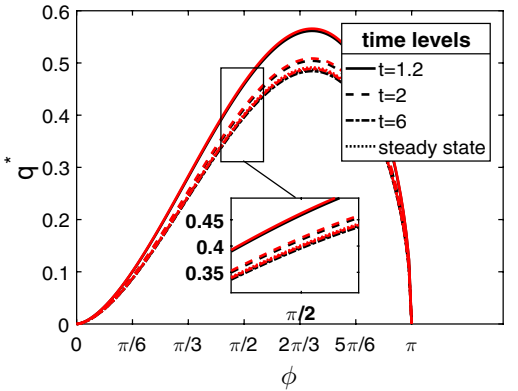

(c) Dilatant Fluid

Fig. 6 Variation of heat flux with $\phi$ for Cylinder: Non-Isothermal case

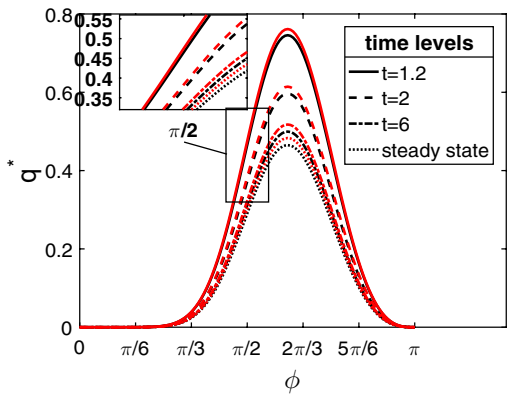

(a) Pseudoplastic fluid

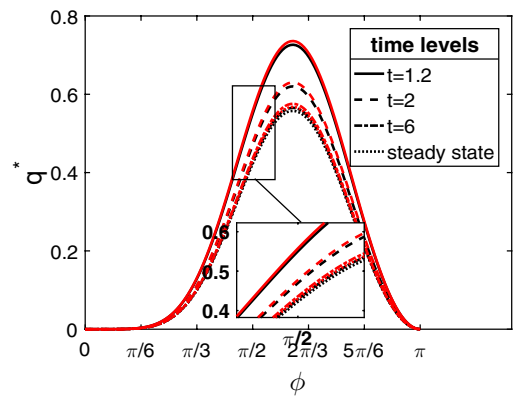

(b) Newtonian Fluid

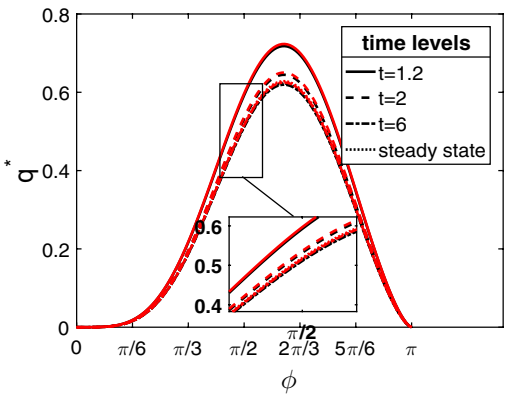

(c) Dilatant Fluid

Fig. 7 Variation of heat flux with $\phi$ for Sphere: Non-Isothermal case

For Figs. 2, 3, 4, 5, 6 and 7, plots in red are for $\gamma=0$ and those in black are for $\gamma=-0.5$. From Fig. 2, the effect of $\gamma$ is noteworthy in the boundary layer only and logarithm scale is used to show the effect of $\gamma$ distinctly. We can observe that for constant viscosity $(\gamma=0)$, the non-dimensional velocity profile decreases continuously. For variable vis$\operatorname{cosity}(\gamma \neq 0)$, the velocity profile attains its maxima in the range $10^{-1}<\eta<1$ and then reduces gradually. The variation of the velocity profile with $\gamma$ is more prominent for a pseudoplastic fluid than for a Dilatant fluid. As we move far from the boundary layer region, the change in value of $\gamma$ doesn't alter the velocity profile as the nanoparticle volume fraction $\Phi$ tend to achieve a constant value $\Phi_{\infty}$ . Figure 3 depicts that the increase in variable viscosity parameter decreases the magnitude of concentration gradient near the wall surface. The effect of $\gamma$ on concentration profile reduces as the value of $N$ is increased from 0.5 to 2 . The local heat flux for a horizontal cylinder and a sphere are given by 
Table 5 Values of reduced Nusselt number $-\theta^{\prime}(0)$ and reduced Sherwood number- $f^{\prime}(0)$ at steady state for vertical plate for isothermal case

\begin{tabular}{|c|c|c|c|c|c|c|c|c|c|c|c|}
\hline \multicolumn{3}{|c|}{$N r=10^{-3}, N t=10^{-6}, N b=10^{-5}$} & \multicolumn{3}{|c|}{$N r=10^{-2}, N b=10^{-4}, \gamma=0$} & \multicolumn{3}{|c|}{$N r=10^{-2}, N t=10^{-6}, \gamma=0$} & \multicolumn{3}{|c|}{$N t=10^{-6}, N b=10^{-5}, \gamma=0$} \\
\hline$\gamma$ & $-\theta^{\prime}(0)$ & $-\omega^{\prime}(0)$ & $N t$ & $-\theta^{\prime}(0)$ & $-\omega^{\prime}(0)$ & $\mathrm{Nb}$ & $-\theta^{\prime}(0)$ & $-\omega^{\prime}(0)$ & $\mathrm{Nr}$ & $-\theta^{\prime}(0)$ & $-\omega^{\prime}(0)$ \\
\hline \multirow[t]{2}{*}{-0.5} & 0.4379 & 14.3623 & $10^{-9}$ & 0.4438 & 17.0600 & $10^{-7}$ & 0.4438 & 16.8933 & $10^{-5}$ & 0.4439 & 17.1071 \\
\hline & $0.4379 *$ & $14.5928^{*}$ & & $0.4437^{*}$ & $17.6831^{*}$ & & $0.4438^{*}$ & $17.5101 *$ & & $0.4439^{*}$ & $17.7387^{*}$ \\
\hline \multirow[t]{2}{*}{-0.1} & 0.4429 & 16.6092 & $10^{-8}$ & 0.4437 & 17.0600 & $10^{-6}$ & 0.4438 & 17.0434 & $10^{-4}$ & 0.4439 & 17.1067 \\
\hline & $0.4428^{*}$ & $17.1597^{*}$ & & $0.4437^{*}$ & $17.6830^{*}$ & & $0.4438^{*}$ & $17.6657^{*}$ & & $0.4439^{*}$ & $17.7381^{*}$ \\
\hline \multirow[t]{2}{*}{-0.05} & 0.4434 & 16.8583 & $10^{-7}$ & 0.4437 & 17.0600 & $10^{-5}$ & 0.4438 & 17.0584 & $10^{-3}$ & 0.4439 & 17.1023 \\
\hline & $0.4434^{*}$ & $17.4518^{*}$ & & $0.4437^{*}$ & $17.6830^{*}$ & & $0.4437^{*}$ & $17.6829 *$ & & $0.4439^{*}$ & $17.7329^{*}$ \\
\hline \multirow[t]{2}{*}{-0.005} & 0.4439 & 17.0782 & $10^{-6}$ & 0.4437 & 17.0599 & $10^{-4}$ & 0.4438 & 17.0598 & $10^{-2}$ & 0.4439 & 17.0583 \\
\hline & $0.4440^{*}$ & $17.7102^{*}$ & & $0.4437^{*}$ & $17.6829 *$ & & $-^{*}$ & $-^{*}$ & & $0.4438^{*}$ & $17.6813^{*}$ \\
\hline
\end{tabular}

$$
\begin{aligned}
q_{c}^{*}= & q_{\mathrm{v}}{ }^{*}(\sin \phi)^{\frac{1}{N}} \\
& \times\left[\frac{\left(\int_{0}^{\phi}(\sin x)^{\frac{1}{N}} \mathrm{~d} x\right)^{\frac{(2 N+1) \lambda-N}{2 N}}}{\left(\int_{0}^{\pi}(\sin x)^{\frac{1}{N}} \mathrm{~d} x\right)^{\frac{(2 N+1) \lambda}{2 N}}}\right], \text { Horizontal Cylinder } \\
q_{\mathrm{s}}^{*}= & q_{\mathrm{v}}^{*}(\sin \phi)^{\frac{N+1}{N}} \\
& \times\left[\frac{\left(\int_{0}^{\phi}(\sin x)^{\frac{2 N+1}{N}} \mathrm{~d} x\right)^{\frac{(2 N+1) \lambda-N}{2 N}}}{\left.\left(\int_{0}^{\pi}(\sin x)^{\frac{2 N+1}{N}} \mathrm{~d} x\right)^{\frac{(2 N+1) \lambda}{2 N}}\right], \text { Sphere }}\right.
\end{aligned}
$$

Figures 4, 5, 6 and 7 represent the variation of heat flux profile with $\phi$ for cylinder and sphere, respectively. The effect of $\gamma$ is most prominent in pseudoplastic fluids which is expected from Table 1 as well. The limiting values are calculated at stagnation points $\phi=0, \pi$ for both sphere and cylinder. For isothermal bodies, this value turns out to be 0 for $N=0.5, q_{\mathrm{v}}{ }^{*}$ for $N=1$ and $\infty$ for $N=2$ at $\phi=0$ and zero at $\phi=\pi$. For $\lambda=1$, the value is zero at both the stagnation points. From Fig. 6 , the maxima value obtained by heat flux in case of cylinder is $\frac{2 \pi}{3}$ for $N=1, \phi<\frac{2 \pi}{3}$ for $N=0.5$ and $\phi>\frac{2 \pi}{3}$ for $N=2$. From Fig. 7, i.e. for sphere, maxima is at the same point for all three types of fluid considered in this study, which is at $\frac{3 \pi}{5}$ approximately.

\section{Conclusion}

The study of the effect of nanoparticle volume fractiondependent viscosity on the free convection heat transfer for an axissymmetric body immersed in non-Newtonian nanofluid is carried out. Keller Box method has been used to solve the dimensionless nonlinear partial differential equations together with dimensionless boundary conditions. The axisymmetric bodies considered in this paper are vertical flat plate, horizontal cylinder and sphere. The variation of reduced Nusselt number and reduced Sherwood number due to varying viscosity parameter is tabulated in this study. The effect of Buoyancy ratio, Brownian motion and thermophoresis on Nusselt number and Sherwood number is analysed in the study. The results are validated using previous studies. The transition of velocity and concentration profile with respect to dimensionless parameter $t$ is graphically presented in the study. We can conclude that the variable viscosity in terms of nanoparticle volume fraction is an important aspect in heat transfer problems, and hence, a combination of nanoparticle volume fraction and temperature should be opted for future studies.

\section{Compliance with ethical standards}

Conflict of interest On behalf of all authors, the corresponding author states that there is no conflict of interest.

\section{References}

1. Menni Y, Chamkha Ali J, Azzi A (2019) Nanofluid transport in porous media: a review. Spec Top Rev Porous Media Int J 10:49-64. https://doi.org/10.1615/SpecialTopicsRevPorousMed ia. 2018027168

2. Sheremet MA, Pop I, Shenoy A (2015) Unsteady free convection in a porous open wavy cavity filled with a nanofluid using Buongiorno's mathematical model. Int Commun Heat Mass Transfer 67:66-72. https://doi.org/10.1016/j.icheatmasstrans fer.2015.07.007

3. Loganathan P, Chand P Nirmal, Ganesan P (2015) Transient natural convective flow of a nanofluid past a vertical plate in the presence of heat generation. J Appl Mech Tech Phys 56:433. https://doi.org/10.1134/S002189441503013X

4. Alsabery Al, Chamkha AJ, Saleh H, Hashim I (2017) Transient natural convective heat transfer in a trapezoidal cavity filled with non-Newtonian nanofluid with sinusoidal boundary conditions on both sidewalls. Powder Technol 308:214-234. https ://doi.org/10.1016/j.powtec.2016.12.025

5. Deka RK, Paul A, Chaliha A (2017) Transient free convection flow past vertical cylinder with constant heat flux and mass 
transfer. Ain Shams Eng J 8:643-651. https://doi.org/10.1016/j. asej.2015.10.006

6. Tilton N (2018) Onset of transient natural convection in porous media due to porosity perturbations. J Fluid Mech 838:129-147. https://doi.org/10.1017/jfm.2017.861

7. Vasu B, Gorla Rama Subba Reddy, Bég O Anwar, Murthy PVSN, Prasad VR, Kadir Ali (2019) Unsteady flow of a nanofluid over a sphere with nonlinear boussinesq approximation. JThermophys Heat Transfer 33:343-355. https://doi.org/10.2514/1.T5516

8. Lai FC, Kulacki FA (1990) The effect of variable viscosity on convective heat transfer along a vertical surface in a saturated porous medium. Int J Heat Mass Transfer 33(5):1028. https://doi. org/10.1016/0017-9310(90)90084-8

9. Pop I, Gorla RSR, Rashidi M (1992) The effect of variable viscosity on flow and heat transfer to a continuous moving flat plate. Int J Eng Sci 3(1):1-6. https://doi.org/10.1016/0020-7225(92)90115 $-\mathrm{W}$

10. Bagai S (2004) Effect of variable viscosity on free convection over a non-isothermal axisymmetric body in a porous medium with internal heat generation. Acta Mech 169:187-194. https://doi. org/10.1007/s00707-003-0069-7

11. Jayanthi S, Kumari M (2007) Effect of variable viscosity on nonDarcy free or mixed convection flow on a vertical surface in a non-Newtonian fluid saturated pourous medium. Appl Math Comput 186:1643. https://doi.org/10.1016/j.amc.2006.08.072

12. El-Amrani Mofdi, Seaïd Mohammed (2008) A finite element modified method of characteristics for convective heat transport. Numer Methods Partial Differ Equ 24(3):776-798. https:// doi.org/10.1002/num.20288

13. Moorthy M, Senthilvadivu K, Kannan T (2017) Free convection in non-newtonian power-law fluids along a vertical plate with variable viscosity and thermal stratification in the presence of internal heat generation. Heat Transf Res 48(1):9-22. https://doi. org/10.1615/HeatTranRes.2016008318

14. Bagai S, Sharma M (2019) A numerical study of transient free convection from an axisymmetric body in a porous media saturated by nanofluid. J Nanofluids 8(6):1345-1354. https://doi. org/10.1116/jon.2019.04.1675

15. Mahbubul IM, Saidur R, Amalina MA (2012) Latest developments on the viscosity of nanofluids. Int J Heat Mass Transf 55:874-885. https://doi.org/10.1016/j.ijheatmasstransfer.2011.10.021

16. Ravisankar B, Tarachand V (2013) Influence of nanoparticlevolume fraction, particle size and temperature on thermal conductivity and viscosity of nanofluids: a review. Int J Automot Mech Eng 8:1316-1338. https://doi.org/10.15282/ijame 8.2013.20.0108

17. Rudyak V Ya, Krasnolutskii SL (2014) Dependence of the viscosity of nanofluids on nanoparticle size and material. Phys Lett $A$ 378:26-27. https://doi.org/10.1016/j.physleta.2014.04.060
18. Bashirnezhad K, Bazri S, Safaei MH, Goodarzi M, Dahari M, Mahian O, Dalkılıça AS, Wongwises S (2016) Viscosity of nanofluids: a review of recent experimental studies. Int Commun Heat Mass Transf 73:114-123. https://doi.org/10.1016/j.icheatmass transfer.2016.02.005

19. Noghrehabadi A, Ghalambaz FM, Ghanbarzadeh A (2014) Effects of variable viscosity and thermal conductivity on natural convection of nanofluids past a vertical plate in porous media. $J$ Mech 30(3):265-275. https://doi.org/10.1017/JPorMedia.v18. i4.10

20. Esfe $M H$, Afrand $M$, Gharehkhani S, Rostamian $H$, Toghraie $D$, Dahari M (2016) An experimental study on viscosity of aluminaengine oil: effects of temperature and nanoparticles concentration. Int Commun Heat Mass Transf 76:202-208. https://doi. org/10.1016/j.icheatmasstransfer.2016.05.013

21. Esfe MH (2018) The investigation of effects of temperature and nanoparticles volume fraction on the viscosity of copper oxide-ethylene glycol nanofluids. Period Polytech Chem Eng 62(1):43-50. https://doi.org/10.3311/PPch.9741

22. Schelfhout AJ, Vafaei $S$ (2019) Experimental investigation of the effects of nanofluid characteristiccs on viscosity. Therm Fluids Eng Conf 4:1027-1037. https://doi.org/10.1615/TFEC2019. fnd.027627

23. Nield DA, Bejan A (2013) Convection in porous media, 4th edn. Springer, New York

24. Einstein A (1906) A new determination of the molecular dimensions. Ann Phys 324(2):289-306

25. Brinkman HC (1952) The viscosity of concentrated suspensions and solutions. J Chem Phys 20(4):571. https://doi. org/10.1063/1.1700493

26. Batchelor GK (1977) The effect of Brownian motion on the bulk stress in a suspension of spherical particles. J Fluid Mech 83(1):97-117. https://doi.org/10.1017/S0022112077001062

27. Keller HB (1970) A New difference scheme for parabolic problems. Numer Solut Partial Differ Equ II:327-350. https://doi. org/10.1016/B978-0-12-358502-8.50014-1

28. Cebeci T, Bradshaw P (1984) Physical and computational aspects of convective heat transfer. Springer, Berlin

Publisher's Note Springer Nature remains neutral with regard to jurisdictional claims in published maps and institutional affiliations. 